\title{
EFFECT OF PULMONARY HYPERTENSION SECONDARY TO CONGENITAL HEART DISEASE ON RIGHT VENTRICULAR VOLUMES AND FUNCTION: A COMPARATIVE STUDY BETWEEN TWO DIMENSIONAL, THREE DIMENSIONAL ECHOCARDIOGRAMS AND INVASIVE RV QUANTIFICATION
}

\author{
Yasmin A. Esmail, Ghada S. EL Shahid, Alaa M. Roushdy and \\ Mohamed R. Hussein.
}

\begin{abstract}
Department of Cardiology Faculty of Medicine - Ain Shams University
\end{abstract}

Corresponding author:

Mohamed R. Hussein.

Mobile: 01002086538

E mail:

Mohamed_rashad 1988@hotm ail.com

Received: $11 / 4 / 2019$

Accepted: 24/4/2019

\begin{abstract}
Background: Quantitative echo cardiographic assessment of right ventricular $(R V)$ function is becoming of increasing interest in cardiac diseases that affect the right ventricle, such as congenital heart disease and acute or chronic pulmonary hypertension (PH),but is still challenging due to $R V$ complex anatomy and structure.
\end{abstract}

Aim of the Work: To study the effect of $\mathrm{PH}$ on the $\mathrm{RV}$ volumes and function using $2 D E, 3 D E$ and $R V$ quantification by angiography in patients with $\mathrm{PH}$ secondary to congenital heart disease and to compare between $3 D R V$ quantification by echocardiogram and invasive by angiography in patients with $\mathrm{PH}$ secondary to congenital heart disease.

Patients and Methods: This cross sectional study included all patients with congenital heart diseases complicated by pulmonary hypertension referred to cardiology department in Ain shams university hospital for elective hemodynamic assessment.

Results: The diagnostic accuracy of $3 D R V$ and angiography (Ang.) $R V$ parameters for detecting hemodynamic signs of pulmonary hypertension (a composite of $P V R>7$ ) was shown by receiver-operating characteristic curves. The parameters with the largest areas under the curve (AUC) were: $3 D$ tenting volume (1.000) had the highest area under the curve followed by mean PAP and PASP, followed by 3D-TV annulus area, Ang. RV EF,3D RV EF And lastly FAC. The best cut-off values of: 3D TV tenting volume $>4.8$ (Se: 100\%, Sp: 100\%), PASP > 80(Se: $100 \%$, Sp: 97.5\%), mean PAP >50 (SE:100, SP: 97.5\%), 3D TV annulus area $>9.3$ (SE: 100\%, SP: 92.5), Ang. $R V E F<=36 \%$ ( $S E=100 \%$, $S P=80 \%$ ), $3 D$ RV EF $<=42 \%$ (SE=100\%, SP+77.5\%)and $R V F A C<=36 \%$ (Se: 100\%, Sp: $67.5 \%)$.

Conclusion: In PH patients, the quantitative assessment of global and regional $R V$ function by $3 D$ provided a useful hemodynamic and prognostic information. Several indices can be obtained by $3 D$ echocardiography non-invasively, to predict the PVR in congenital heart disease with $\mathrm{PH}$.

Key words: Pulmonary hypertension, congenital heart disease, right ventricle, two-dimensional, three-dimensional echocardiograms.

\section{INTRODUCTION:}

In understanding the progression of right ventricular (RV) failure in pulmonary arterial hypertension (PAH), a key knowledge is the mechanisms that underlie the transition from normal RV function to $\mathrm{RV}$ dysfunction and failure, and how these transitions are dependent on changes in 
pulmonary vascular function as the disease progresses. Clinical studies have shown that elevated pulmonary vascular resistance correlates with mortality in $\mathrm{PAH}^{1-3}$.

Cardiac magnetic resonance is currently the most reliable non-invasive tool for this purpose, mostly due to its unique spatial resolution and 3D imaging capacity. Cardiac magnetic resonance is, however, inapplicable in certain patients (e.g. with claustrophobia or implanted metallic devices). In addition, its wide use is precluded by cost, long scan and analysis time, limited availability, and dedicated expertise. Moreover, CMR has limited ability to assess load-independent parameters (such as myocardial contractility) in patients with long-standing pressure overloaded RV.

Accurate volume analysis independent of RV size and shape, without foreshortened views and geometric assumptions ensure the superiority of RT3DE for RV quantitation over the conventional echocardiography methods. Good correlation between RT3DE and CMR for calculating RV volumes and EF was reported. Compared with CMR data, RV volumes calculated from 3D echocardiography showed significantly better agreement and lower intra- and inter observer variability than $2 \mathrm{D}$ echocardiography.

Right heart catheterization is the gold standard method for diagnosis of pulmonary hypertension and an important tool for testing the eligibility of patients with CHD to undergo operations for correction of heart defects $^{5-13}$. PVR is routinely calculated using catheterization-derived measurements - as the ratio of the transpulmonary pressure gradient $(\Delta \mathrm{p})$ to flow $(\mathrm{Qp})^{3,4}$ The invasive nature of this measurement might increase patient's risk and therefore, tends to restrict the frequency with which it is obtained. As newer therapies for $\mathrm{PH}$ emerge and cardiothoracic surgeons accept patients with higher and higher PVR, a noninvasive method that would allow more frequent measurements of PVR may prove useful ${ }^{2,3,4}$.

\section{AIM OF THE WORK:}

1. To study the effect of $\mathrm{PH}$ on the RV volumes and function using $2 \mathrm{De}, 3 \mathrm{DE}$ and RV quantification by angiography in patients with $\mathrm{PH}$ secondary to congenital heart disease.

2. To Compare between 3D RV quantification by echocardiogram and invasive by angiography in patients with PH secondary to congenital heart disease.

- Type of Study: cross sectional study

- Study Period: over a period from 2017-2018

\section{- Study Population :}

\section{Inclusion Criteria:}

The study included all patients with congenital heart diseases complicated by pulmonary hypertension referred to cardiology department in Ain shams university hospital for elective hemodynamic assessment.

\section{- Exclusion Criteria:}

- Subjects with other conditions that can cause pulmonary hypertension, prior surgery that required operative manipulation of the RV, cardiomyopathy, arrhythmias, comorbidities, insufficient ultrasound image quality (defined as more than 3 myocardial segments that were not optimally visualized using conventional $2 \mathrm{DE}$ ), any contraindication to catheterization, any contraindication to general anesthesia and any complex congenital heart disease rather than simple intracardiac shunts

- Sample Size: all patients with congenital heart diseases complicated by pulmonary hypertension referred to 
cardiology department in Ain shams university hospital for elective hemodynamic assessment over a period from 2017-2018.

\section{- Ethical Considerations:}

- Approval of Ain Shams University ethical committee

- Informed consent was obtained from all patients.

- All the patients in the master table were coded by numbers to preserve their confidentiality and no one has right to read the patient medical information except the main researcher.

- Study Procedures: all procedures were carried out by the most competent personal in our institution

\section{A. Before catheterization:}

* Two dimensional Echocardiography:

Transthoracic echocardiographic examination using a Vivid 5, Vivid E9 or S5 machine with an M4S matrix sector array probe with a frequency of $2.5 \mathrm{MHZ}$ (General Electric Vingmed Ultrasound, Horten, Norway). M-mode, two dimensional, tissue Doppler as well as pulsed and continuous Doppler flow across the different heart valves in all the standard views are to be done with particular emphasis on:

I. RV fractional area change( $R V F A C)$.

II. Tricuspid annular plane systolic excursion (TAPSE).

III. Pulmonary artery systolic pressure $(P A S P)$ and mean pulmonary artery pressure

IV. Different congenital heart diseases like ventricular septal defect, Patent ductous arteriosus, Atrialseptal defect, common AV canal.

* Three dimensional Echocardiography:

3DE was done using a dedicated wide-angle, broadband 4V (GE E95) matrix-array transducer to allow for full cover of the entire RV by the pyramidal volume, with special attention to the outflow tract and upper anterior wall.

After offline analysis all the following data was obtained:3D EDV, 3D ESV, 3D EF,3D Stroke volume indexed ,3D Model of RV, 3D Tricuspid valve area and 3D TV tenting volume.

\section{B. Catheterization:}

* The pulmonary vascular resistance PVR in Wood units (WU) will be calculated.

* RV quantification by angiography:

For calculation of angiographic volumes, the thirty-degree right anterior oblique (RAO) view will be utilized. The diastolic and systolic images will be selected, and a contour drawn. The area within the contour will be then calculated.

\section{Statistical Analysis:}

Data are going to be presented as mean \pm SD. Regression analysis are to be used to relate Doppler parameters to hemodynamics. Significance will be set at a $p$ value $\leq 0.05$.

\section{RESULTS}

Patient demographic and baseline data:

50 patients with simple congenital heart disease complicated with $\mathrm{PH}$ were included in the study. One patient was excluded due to in accurate measurement of $3 \mathrm{D} R \mathrm{RV}$ volumetric analysis (unsatisfactory echocardiographic window due to missing the anterior wall of the RV). The final study comprised 49 patients (Female $=30$ patient $\mathrm{s}$, Males $=19$ patients) as shown in figure (1)

Demographic characteristics of 49 patients were shown in table (1). 
Yasmin A. Esmail, et al.,

Table (1):

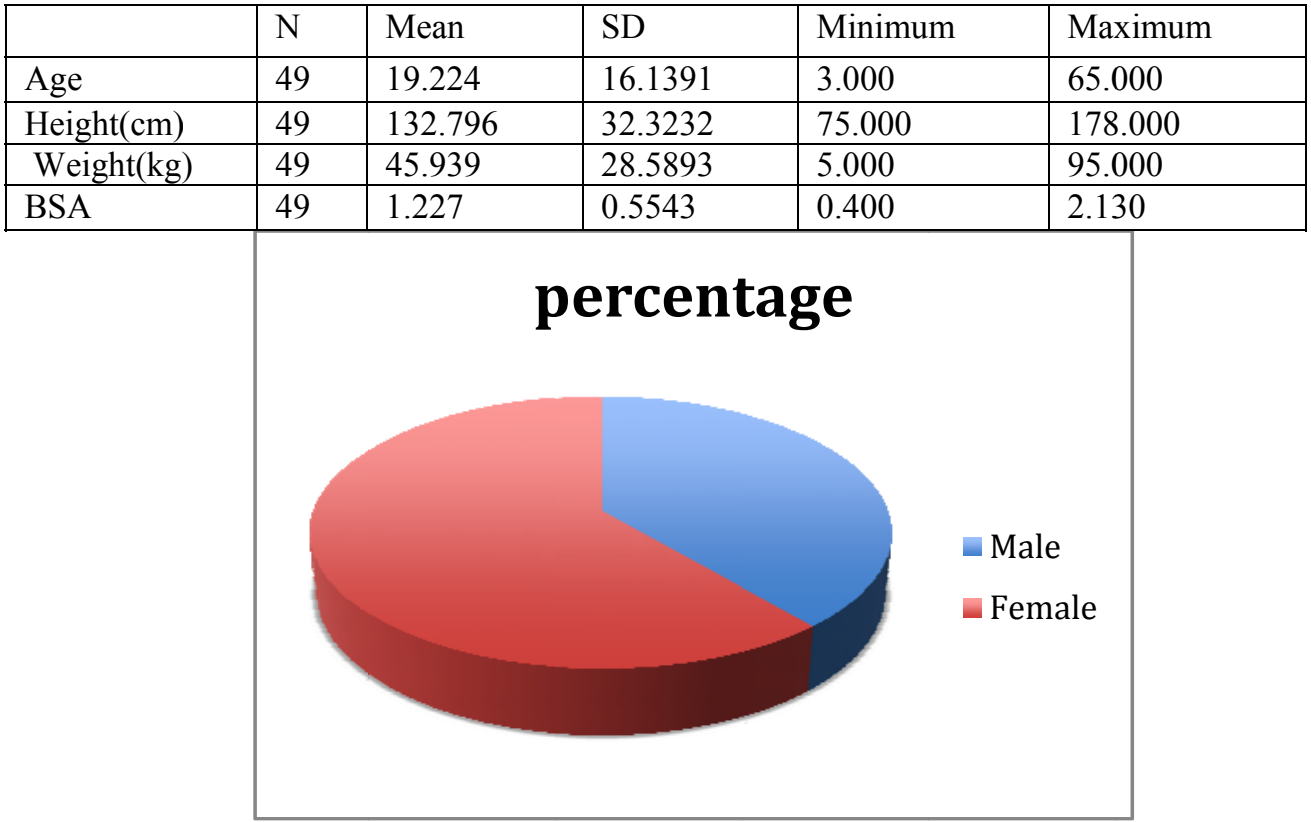

Figure (1): Pie chart demonstrates the percentage of both genders in the study group.

The patients were classified in to subgroups according the underlying congenital defectas shown in figure (2):ASD $(n=20,40.8 \%)$, PDA $(n=12,24.5 \%)$, VSD $(n=13,26.5 \%), \operatorname{CAVCD}(n=3,6.1 \%)$, $\operatorname{TAPVD}(\mathrm{n}=1,2 \%)$

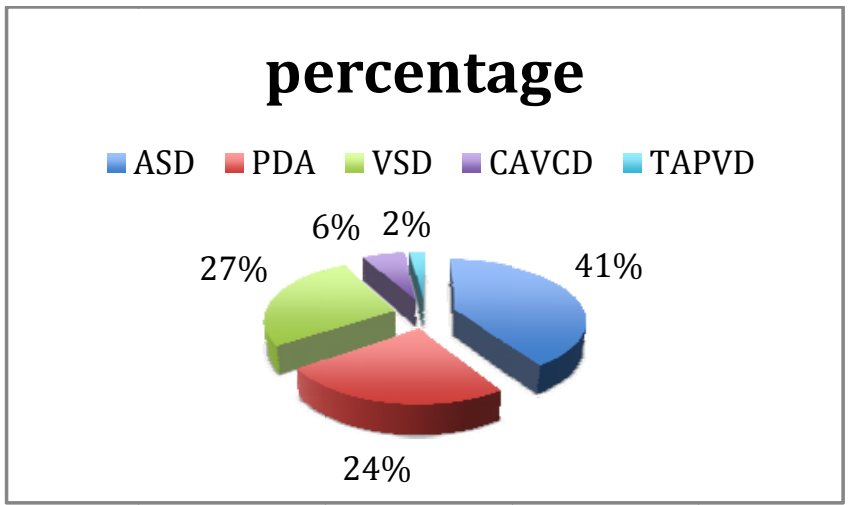

Figure (2): Piechart demonstrates the subgroups of the study population.

Considering the entire study population, real time 3D images were successfully analysed in 49 patients as shown in table (2).

Table (2):

\begin{tabular}{|l|l|l|l|l|l|l|}
\hline & $\mathrm{N}$ & Mean & SD & Median & Minimum & Maximum \\
\hline 3D,RV ESV $(\mathrm{ml} / \mathrm{m} 2)$ & 49 & 47.245 & 29.8430 & 41.000 & 17.000 & 194.000 \\
\hline 3D,RVEDV $(\mathrm{ml} / \mathrm{m} 2)$ & 49 & 82.449 & 42.7157 & 75.000 & 35.000 & 303.000 \\
\hline 3D SV i & 49 & 35.184 & 15.0813 & 33.000 & 15.000 & 109.000 \\
\hline 3D TV area & 49 & 6.647 & 3.9727 & 4.700 & 1.700 & 18.600 \\
\hline 3D RVEF & 49 & 43.216 & 8.1895 & 46.000 & 26.700 & 58.500 \\
\hline 3D Tricuspid tenting volume & 49 & 3.118 & 2.2258 & 2.200 & 0.600 & 9.500 \\
\hline
\end{tabular}

Angiography RV volumes quantification were successfully analysed in 49 patientsas shown in table (3). 
Table (3)

\begin{tabular}{|l|l|l|l|l|l|}
\hline & $\mathrm{N}$ & Mean & SD & Minimum & Maximum \\
\hline Angiography SVi & 49 & 40.849 & 17.8290 & 15.000 & 90.000 \\
\hline Angiography RVEDV (ml/m2) & 49 & 107.414 & 58.7876 & 35.000 & 334.000 \\
\hline PVR (woods) & 49 & 4.758 & 3.0539 & 1.300 & 13.400 \\
\hline Angiography RVEF & 49 & 40.198 & 11.0507 & 21.000 & 66.000 \\
\hline Angiography RVESV (ml/m2) & 49 & 66.435 & 46.5777 & 13.000 & 244.000 \\
\hline
\end{tabular}

Conventional 2D echocardiography parameters of RV were successfully analysed in 49 patients as in table (4).

Table (4)

\begin{tabular}{|l|l|l|l|l|l|}
\hline & $\mathrm{N}$ & Mean & SD & Minimum & Maximum \\
\hline Mean PAP(mmhg) & 49 & 33.633 & 23.8036 & 10.000 & 98.000 \\
\hline PASP(mmhg) & 49 & 51.796 & 39.2242 & 13.000 & 156.000 \\
\hline RV FAC & 49 & 36.920 & 10.4570 & 15.200 & 54.200 \\
\hline TAPSE $(\mathrm{mm})$ & 49 & 17.92 & 5.4766 & 3.000 & 28.000 \\
\hline
\end{tabular}

Correlation figures:

We compared 3D RV parameters, Angiography RV parameters with PVR, we found that both of them are statistically correlated with PVR, however 3D RV EF has a stronger linear correlation with PVR than Ang. RV EF depending on $r$ value (3D RV EF $r=-0.666 ; p<0.001)$, angiography performance $(\mathrm{r}=-0.588 ; \mathrm{p}<0.001)$ figures $(4,5)$, both of them are far better than $3 \mathrm{D}$ RV volumes and angiography $\mathrm{RV}$ volumes with PVR.

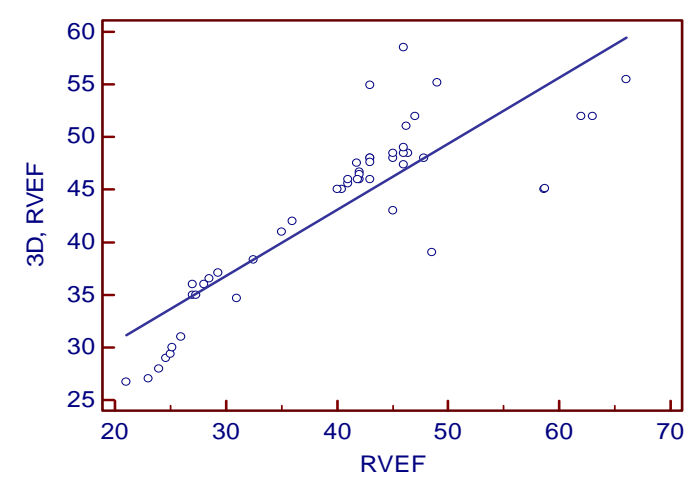

Figure (3): Significant correlation between Angiography RV EF and 3D RV EF
The RV EF and volumes measured by 3D echocardiography and quantified by angiography were compared with each other, a strong linear correlation were detected between:

3D RV EF and angiography RV EF ( $r=$ $0.847 ; \mathrm{p}<0.001)$, Figure (3).

3D RV EDV and angiography RV EDV $(\mathrm{r}=0.817 ; \mathrm{p}<0.001)$,

The same between 3D RV ESV and Angiography RV ESV ( $\mathrm{r}=0.870 ; \mathrm{p}<$ 0.001).

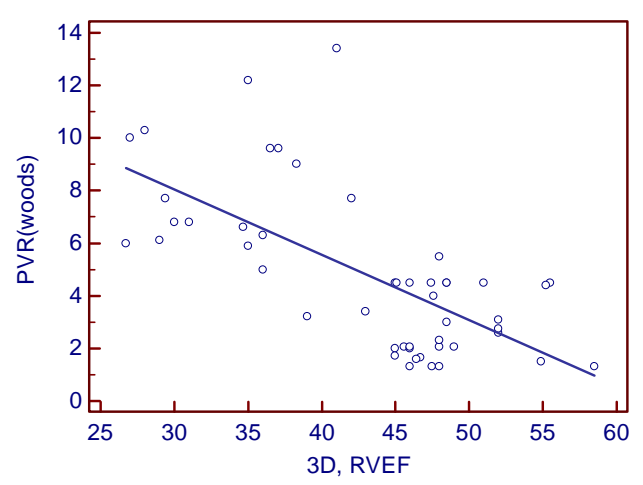

Figure (4): correlation between 3D RV EF and PVR 


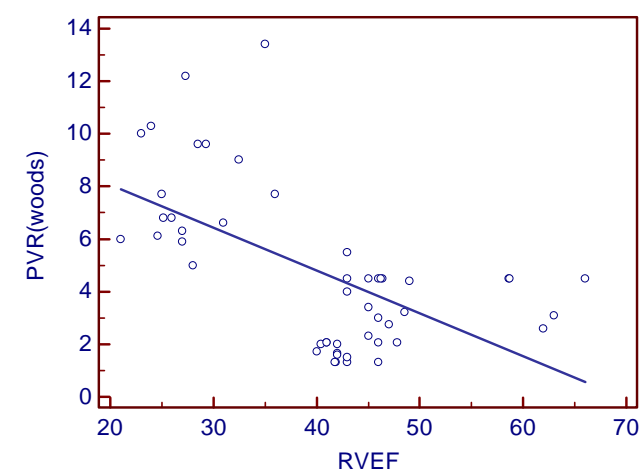

Figure (5): correlation between Angiography RV EF and PVR

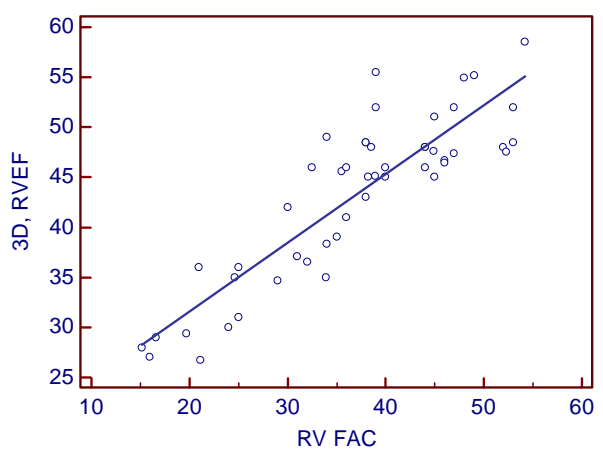

Figure (6): correlation between 3D RV EF and RV FAC

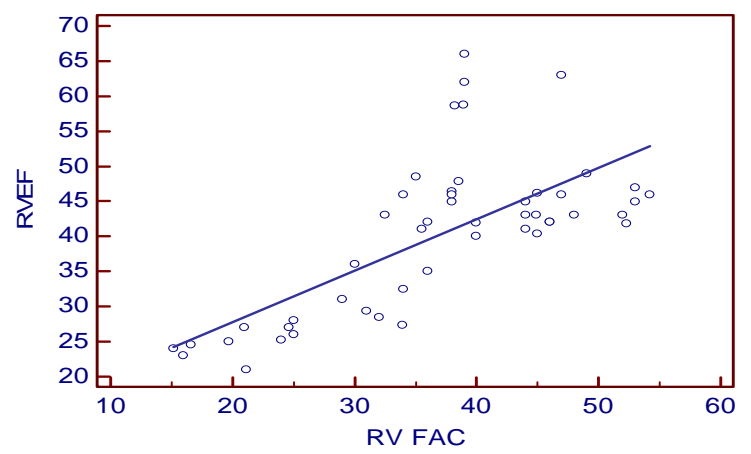

Figure (7): correlation between Ang. RVEF and FAC

3D and conventional 2D echocardiography parameters were compared with each other) where 3D RV EF was strongly correlated with PASP and mean PAP in negative manner than Angiography RV EF (3D RV EF with PASP $r=-0.755$, 3D RV EF with PAPm, $r=-0.729$ ) (Ang. RV EF with PASP, $r=-0.697$, Ang. RV EF with PAPM, $r=-0.681)$.

Convention function parameters of RV in terms of RV FAC and TAPSE were compared with 3D RV EF. We observed a strong linear positive correlation between 3D RV EF and FAC ( $r=0.879)$ figure (6) than 3RVEF with TPASE $(r=0.577)$, similar correlation was found between this conventional parameter and Angiography RV EF figure (7).

PVR showed a negative significant correlation with RV FAC ( $\mathrm{r}=-0.617$, $\mathrm{p}<0.0001$ ), however no correlation was detected between it and TAPSE ( $\mathrm{r}=$ -
$0.185, p=0.2043$ ) Analysis of 3 subgroups showed the following correlation: Figure (8)

Both PDA and VSD subgroups showed a strong negative linear correlation between 3D and Ang. RV EF with PVR (VSD 3D RV EF $r=-0.708$, Ang. RV EF, $r=-0.745$ ) (PDA subgroup 3D RV EF, $\mathrm{r}=-0.717$, Ang. RV EF, $r=-0.722$ ), while the ASD subgroup approached a weak negative correlation between the 3D RVEF and Ang. RV EF with PVR (ASD 3D RV EF r $=-0.412$, Ang. RV EF $r=-0.295$ ).

RV EDV and RV ESV measured by the 3D echocardiography and angiography showed strong positive correlation among the 3 subgroups $(\mathrm{r}=0.9)$.

Among the conventional 2D RV parameters between the 3 subgroups, the RV FAC showed strong negative correlation with PVR in the VSD and PDA subgroups only ( $\mathrm{r}=-0.689, / \mathrm{r}=-0.639$ respectively). 


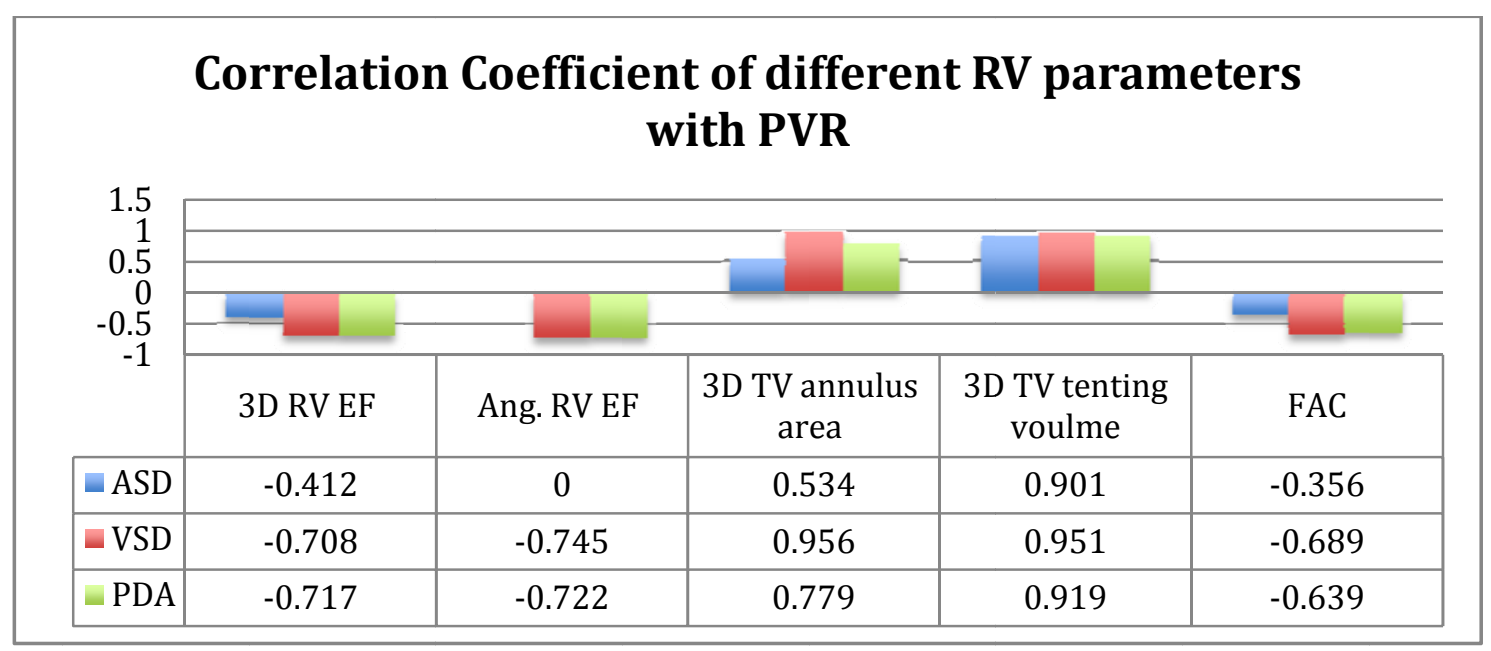

Figure (8): Correlation Coefficient of different RV parameters with PVR

\section{DISCUSSION:}

Our report differs from previous literature in some respects. First, we analysed comparatively the diagnostic accuracy of 3D RV EF and RV EF by angiography, showing a better association with hemodynamic variables reflecting pulmonary hypertension than standard echo indices. To the best of our knowledge, this is the first study assessing the relation between these echocardiographic and angiographic indices with the hemodynamic parameters indicative of pulmonary hypertension in congenital heart disease, this finding showed an additional value compared to standard echo parameters, since the current guidelines point out the importance of early diagnosis of $\mathrm{PH}$ and $\mathrm{RV}$ failure.

Despite the fact that medical therapies reduce PVR, the prognosis of patients with PAH is still poor. The primary cause of death is right ventricular (RV) failure. One possible explanation for this apparent paradox is the fact that a reduction in PVR is not automatically followed by an improvement in RV function and this can be evident by Van de Veerdonk MC, et al, $2011^{(14)}$ where after PAH-targeted therapy, $\mathrm{RV}$ function can deteriorate despite a reduction in PVR. Loss of RV function is associated with a poor outcome, irrespective of any changes in PVR. So early prediction of RV failure will initiate early targeted treatment and improve the outcomes regardless PVR.

In Our study we detected a strong correlation between 3D RV and angiography RV parameters( $\mathrm{EF}$ and volumes), This concept was proven in a different manner by Andrew N, et, al 1988 , ${ }^{(15)}$ who proved a strong correlation between the RV volume cast measured by fluid displacement and that measured by biplane angiography .

Our study has shown that there is no statistically significant correlation between the $3 \mathrm{D}$ and angiography $\mathrm{RV}$ volumes with PVR. This is due to the fact that RV volumes is not solely affected by the pressure overload in congenital intracardiac shunts with pulmonary hypertension, other factors contribute to enlargement of RV volumes for example the additional volume overload in ASD patients.

This was endorsed by, Pei-Ni Jone, et al, $2017^{(16)}$ they conducted a study to evaluate right ventricular functional indices using 3D echocardiography (3DE) between normal children and paediatric pulmonary hypertension $(\mathrm{PH})$ patients, and to evaluate 
these indices as outcome predictors in children with $\mathrm{PH}$.

The study showed that PH patients had higher RV volumes, lower RV EF and this was similar to our study, They concluded that An EDVi $\geq 102 \mathrm{~mL} / \mathrm{m} 2, \mathrm{ESVi} \geq 54$ $\mathrm{mL} / \mathrm{m} 2, \mathrm{SVi} \leq 37 \mathrm{~mL} / \mathrm{m} 2,3 \mathrm{D}$. $\mathrm{EF} \leq 43 \%$ were significantly associated with increased risk of adverse clinical events. However, in our study, we could not obtain neither a significant correlation nor cut off values for $\mathrm{RV}$ volumes and indexed stroke volume in relation high PVR.

Moreover, this is the first study to use the values of $3 \mathrm{D}$ RV EF, 3D TV annulus area , 3D TV tenting volume and angiography RV EF to predict $\mathrm{PVR}>7$ woods in patient with intracardiac shunts complicated with pulmonary hypertension .

On the other hand, Antoniviteralli, et $\mathrm{al}^{(17)}$, had conducted study in 2015 to evaluate the three-Dimensional Echocardiography and 2D-3D Speckle-Tracking Imaging in Chronic Pulmonary Hypertension due to different aetiologies and not congenital heart dieses in particular, they detected a cut off value of 3D RVEF to predict the early RV failure rather than the PVR as obtained in our study

\section{Conclusions:}

Based on the results of the current study it can be concluded that:

- In PH patients, the quantitative assessment of global and regional RV function by $3 \mathrm{D}$ provided us useful hemodynamic and prognostic information.

- Several indices can be obtained by 3D echocardiography non-invasively, predict the PVR in congenital heart disease with pulmonary HTN.

- This is the first study to define the Role of RV quantification by angiography and it is strong correlation with 3D RV indices.

\section{REFERENCES:}

1. Alexis Harrison, Nathan Hatton, John J. Ryan, " The Right Ventricle Under Pressure: Evaluating The Adaptive And Maladaptive Changes In The Right Ventricle In Pulmonary Arterial Hypertension Using Echocardiography", Pulm Circ, 2015, 5:29-47.

2. B. Wang, Y. Feng1, L.-Q. Jia, Et Al, "Accuracy Of Doppler Echocardiography In The Assessment Of Pulmonary Arterial Hypertension In Patients With Congenital Heart Disease",European Review for Medical and Pharmacological Sciences, 2013, 17: 923-928.

3. SaitDemirkol, ŞevketBalta, "Right ventricular functions in pulmonary embolism",AnadoluKardiyolDerg, 2012, 12: 666-7.

4. Alaa Mahmoud Roushdy, Iman Ragab, Wessam Abd el Raouf, " Noninvasive Assessment Of Elevated Pulmonary Vascular Resistance In Children With Pulmonary Hypertension Secondary To Congenital Heart Disease: A Comparative Study Between Five Different Doppler Indices ", J Saudi Heart Assoc, 2012, 24:233-241.

5. YaserJenab, Neda Ghaffari-Marandi, Azam Safir, et al, "Sex-Related Changes In Tissue Doppler Imaging Parameters Among Patients Withacute Pulmonary Thromboembolism ", J Ultrasound Med, 2013, 32:1997-2005.

6. KettiDalla, Caroline Hallman, odd BachHanssen, et al, "Strain Echocardiography Identifies Impaired Longitudinal Systolic Function In Patients With Septic Shock And Preserved Ejection Fraction", Cardiovascular Ultrasound, 2015; 13-30.

7. Gloria Tamborini, Manuela Muratori, Denise Brusoni et al, "Is Right Ventricular Systolic Function Reduced After Cardiac Surgery? A Two- And Three-Dimensional Echocardiographic Study", European 
Journal of Echocardiography 2009: 10; 630 $-634$

8. JesperKjaergaard, Claus Leth Petersen, Andreas Kjaer, et al, "Evaluation Of Right Ventricular Volume And Function By 2D And 3D Echocardiography Compared To MRI", Eur J Echocardiography 2006, 7: 430-438.

9. Mohammed H. Alghamdi, Luc Mertens, Whal Lee, et al, " Longitudinal Right Ventricular Function Is A Better Predictor Of Right Ventricular Contribution To Exercise Performance Than Global Or Outflow Tract Ejection Fraction In Tetralogy Of Fallot: A Combined Echocardiography And Magnetic Resonance Study", European Heart Journal - Cardiovascular Imaging 2013,14: 235 239.

10. Diego Bellavia, Patricia A. Pellikka, Angela Dispenzieri, et al, " Comparison Of Right Ventricular Longitudinal Strain Imaging, Tricuspid Annular Plane Systolic Excursion, And Cardiac Biomarkers For Early Diagnosis Of Cardiac Involvement And Risk Stratification In Primary Systematic (AL) Amyloidosis: A 5-Year Cohort Study", European Heart Journal Cardiovascular Imaging 2012, 13: 680 689.

11. Michal Kidawa, Krzysztof Chizyn ski, MarzennaZielinska, et al, " Real-Time 3D Echocardiography And Tissue Doppler Echocardiography In The Assessment Of Right Ventricle Systolic Function In Patients With Right Ventricular Myocardial Infarction",European Heart Journal Cardiovascular Imaging, 2013, 14: 10021009

12. Heleen B. van der Zwaan, Marcel L. Geleijnse, et al, "Right Ventricular Quantification In Clinical Practice: TwoDimensional Vs. Three Dimension alechocardiography Compared With Cardiac
Magnetic Resonance Imaging", European Journal of Echocardiography 2011,12:, 656-664.

13. Sima R., Bahram L., Mohammad-Reza M., et al, "Pulse Tissue Doppler Analysis Of Tricuspid Annular Motion In Iranian Children", The International Journal of Cardiovascular Imaging (2006) 22: 363367

14. Van de Veerdonk MC, Kind T, Marcus JT, et al . Progressive right ventricular dysfunction in patients with pulmonary arterial hypertension responding to therapy. J Am CollCardiol. 2011;58:25112519

15. Andrew n redington, Huonhgray, Margaret e hodson, Et Al. Characterisation Of The Normal Right Ventricular Pressure-Volume Relation By Biplane Angiography And Simultaneous Micromanometer Pressure Measurements From The Cardiac Department, Brompton Hospital, London, $\mathrm{Br}$ Heart J 1988;59:23-30.

16. Pei-Ni Jone, Michal Scha"fer, Zhaoxing Pan, et al, 3D echocardiographic evaluation of right ventricular function and strain: a prognostic study in pediatric pulmonary hypertension, Pediatric Cardiology, Department of Pediatrics, Children's Hospital Colorado, University of Colorado, European Heart Journal - Cardiovascular Imaging, Volume 19, Issue 9, September 2018, Pages 1026-1033.

17. Antonio Vitarelli, MD, Enrico Mangieri, MD, Claudio Terzano, MD, et al,, Three Dimensional Echocardiography and 2D-3D Speckle-Tracking Imaging in Chronic Pulmonary Hypertension: Diagnostic Accuracy in Detecting Hemo-dynamic Signs of Right Ventricular (RV) Failure, J Am Heart Assoc . v.4 (3); 2015 Mar, PMC4392438. 
تأثير ارتفاع ضغط الدم الثرياني الرئوي بسبب أمراض القلب الخلقية على احجام البطين الأيمن والوظيفة :

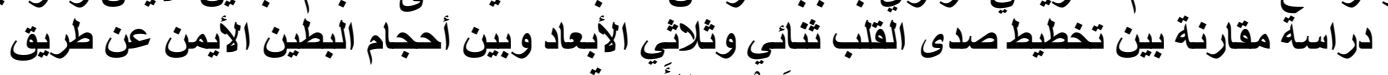

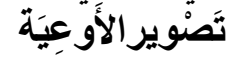

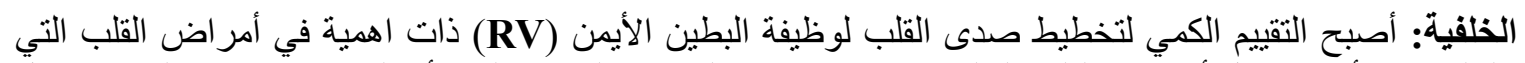

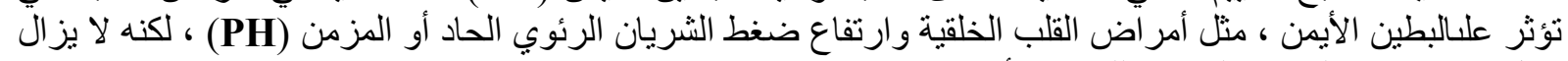

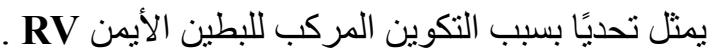

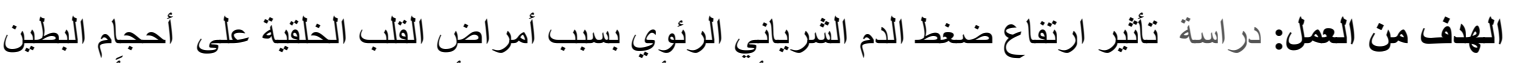

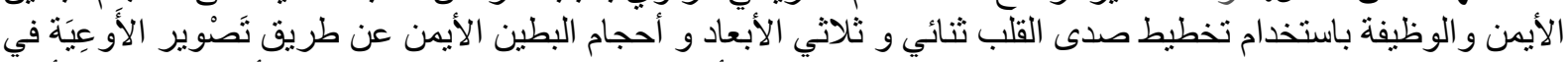

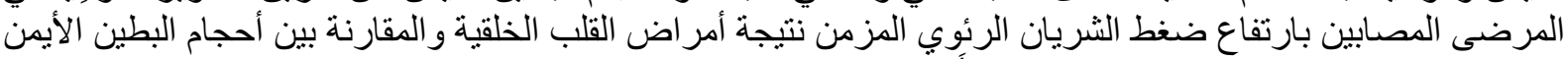

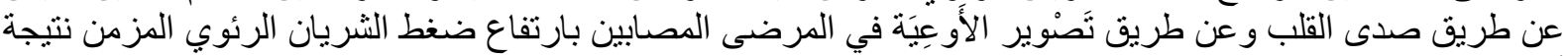
أمر اض طريف القلب الخلقية.

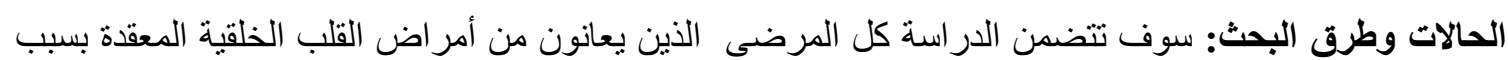

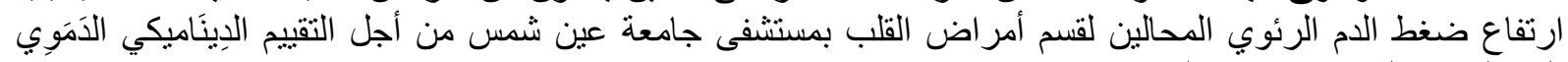

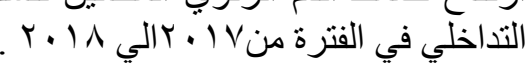

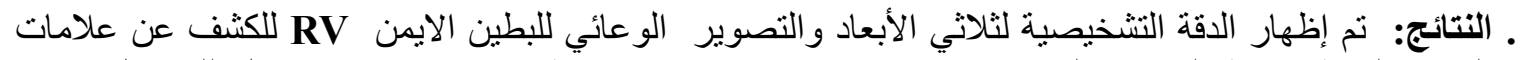

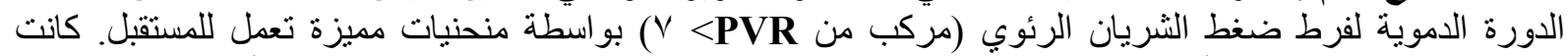

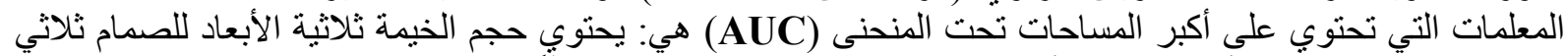

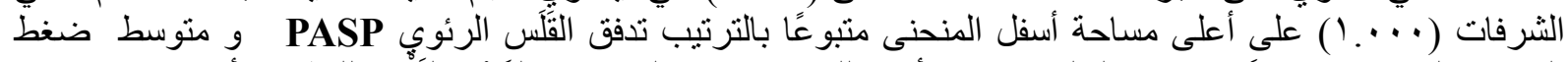

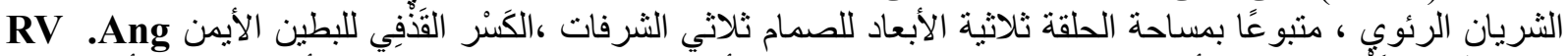

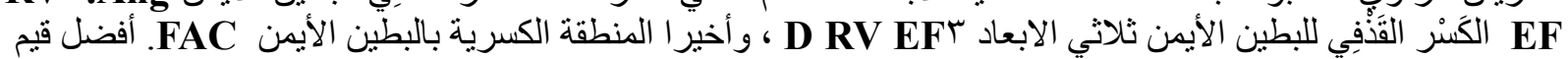

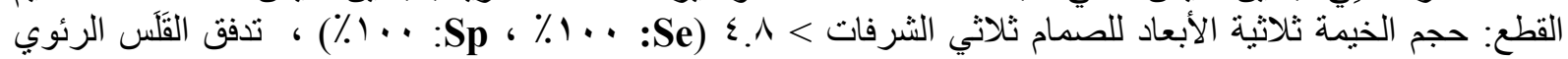

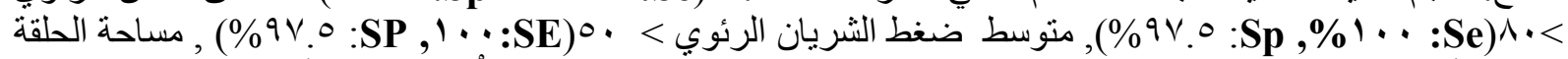

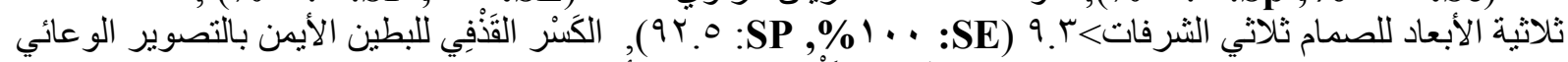

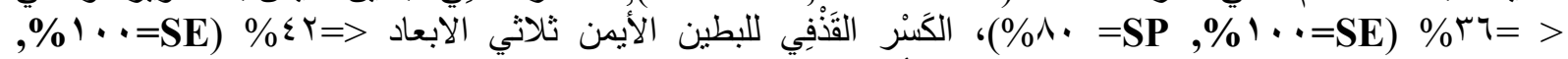

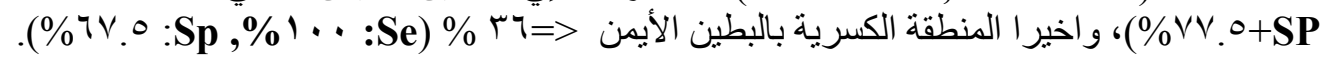

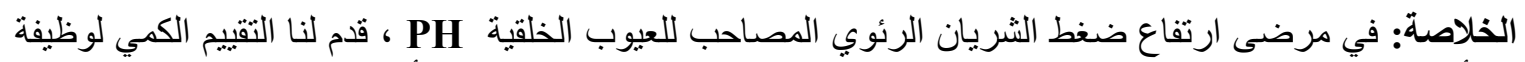

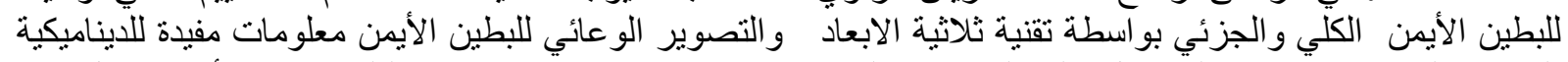

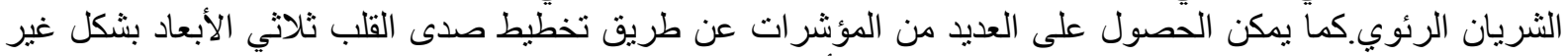

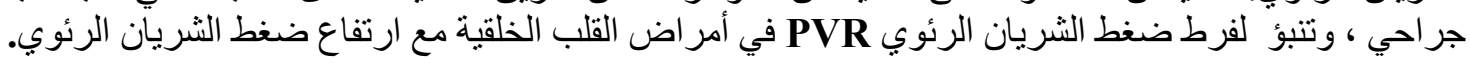

